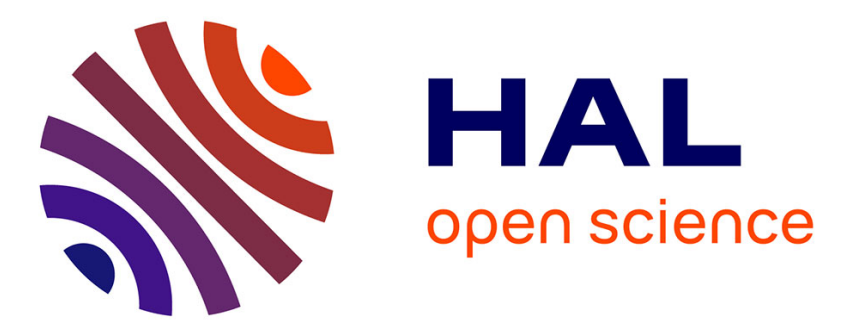

\title{
Speeding Towards Individualized Treatment for Pancreatic Cancer by Taking an Alternative Road Juan Iovanna, Nelson J Dusetti
}

\section{To cite this version:}

Juan Iovanna, Nelson J Dusetti. Speeding Towards Individualized Treatment for Pancreatic Cancer by Taking an Alternative Road. Cancer Letters, 2017, 410, pp.63 - 67. 10.1016/j.canlet.2017.09.016 . hal-01790724

\section{HAL Id: hal-01790724 \\ https://hal.science/hal-01790724}

Submitted on 13 May 2018

HAL is a multi-disciplinary open access archive for the deposit and dissemination of scientific research documents, whether they are published or not. The documents may come from teaching and research institutions in France or abroad, or from public or private research centers.
L'archive ouverte pluridisciplinaire HAL, est destinée au dépôt et à la diffusion de documents scientifiques de niveau recherche, publiés ou non, émanant des établissements d'enseignement et de recherche français ou étrangers, des laboratoires publics ou privés. 


\title{
Speeding Towards Individualized Treatment for Pancreatic Cancer by Taking an Alternative Road
}

\author{
Juan lovanna ${ }^{1}$ and Nelson Dusetti ${ }^{1}$
}

${ }^{1}$ Centre de Recherche en Cancérologie de Marseille (CRCM), INSERM U1068, CNRS UMR 7258, Aix-Marseille Université and Institut Paoli-Calmettes, Parc Scientifique et Technologique de Luminy, Marseille, France.

The authors disclose no conflicts of interest.

Keywords: individualized medicine, pancreatic cancer, PDX, chemograms, molecular signatures, drug sensitivity, tumor heterogeneity.

Corresponding author: Email: juan.iovanna@inserm.fr; Tel: (33) 491 828803; Fax: (33) 491 82886083 


\begin{abstract}
Accumulation of genetic mutations drives the development of pancreatic ductal adenocarcinoma (PDAC). Contrary to what it is expected, however, genetic analyses, no matter how precise or detailed, do not allow the identification of patient groups with different clinical outcomes or the selection of specific treatments. In fact, clinical outcome and sensitivity to treatments are associated with a given phenotype and are therefore associated at a transcriptomic level. In practical terms, therefore, the most appropriate readout for phenotypically stratifying PDACs should be transcriptomic and not genetic analysis. Recently data indicate that studying the expression of a selected gene set could inform selection of the most appropriate treatment for patients, moving towards an individualized medicine approach for this dismal disease. We are optimizing this approach by developing a platform based on obtaining organoids directly from surgical as well as endoscopic ultrasound-guided fine needle aspiration (EUS-FNA) biopsies of tumors, which serve as a source of RNA, allowing determination of the transcription level of some informative genes. We are convinced that in the near future, the treatment of cancers will be preceded by an extensive molecular characterization of cancer cells in order to select the most appropriate treatments.
\end{abstract}




\section{Introduction}

Over recent decades, detailed genetic analysis of tumors has resulted in the identification and validation of crucial genes that are mutated and dysregulated in a tumor-specific manner, indicating a genetic dependency in the development of these tumors, and suggesting that it could be possible to take advantage of these mutations as potential therapeutic targets, where specific drugs are available. Unfortunately, after a highly enthusiastic period, we must recognize that these type of targets can be effectively utilized for only a small percentage of patients; firstly, because relevant drugs are not available, and secondly, because the mutated genes are not druggable. This is the case for pancreatic ductal adenocarcinoma (PDAC), in which the mutations are relatively conserved between tumors (KRAS, P53, SMAD4, CDKN2A, MLL3, TGFBR2, ARID1A, and SF3B1) but targetable genes remain extremely rare since some of them does not present direct enzymatic activity to be inhibited or because their protein-protein interaction-based activity remain technically unattainable for the moment. Alternative strategies must therefore be developed to treat these patients more effectively by using for example the promising genome editing CRISPRCas9-based strategies for targeting to the activating punctual mutations on oncogenes.

\section{Pancreatic ductal adenocarcinoma is a highly heterogeneous disease}

PDAC is one of the most lethal human malignancies and a major health problem, causing around 350,000 deaths per year worldwide [27]. The prognosis is poor, with around $5 \%$ of patients alive at 5 years after diagnosis [27]. Almost all recent phase II and III clinical trials implemented in unselected PDAC populations showed no robust survival benefits, probably because they were tested in unselected PDAC populations that were highly heterogeneous $[18,23,30]$. In fact, a major impediment to the effective treatment of PDAC is the molecular heterogeneity of the disease, reflected in diverse clinical response patterns to therapy. This heterogeneity is shown by the heterogeneous evolution observed in patients with PDAC, with survival that can range from 2-3 months to more than 5 years after diagnosis, and with a strong difference in susceptibility to treatment with classical as well as novel drugs. This may be explained by the fact that each PDAC is controlled by a combination of several modifications of intracellular pathways, which will result in variable susceptibility to drugs, metastasis development, and therefore survival [9, 17, 31]. Furthermore, as the extensive inter- and intratumoral heterogeneity is further characterized, "virtual microdissection" of PDAC transcriptome data has not only allowed identification of tumor subtypes, but also two distinct stromal types [21]. Unfortunately, until now no proposed treatments have taken into account this heterogeneity. In fact, the drugs received by patients suffering from PDAC are chosen according to their general performance status and the stage of their disease. No study of the tumor can predict its responsiveness to the treatment, nor give a prognosis to the disease progression. For example, objective response rates of $31.6 \%$ in FOLFIRINOXtreated patients and $9.4 \%$ in patients treated with gemcitabine have been reported, showing 
that around $70 \%$ and $90 \%$ of patients are non-responders, respectively [6, 8]. But who are these patients? How these patients could be identified? It appears evident that to increase treatment effectiveness, patients should be selected to receive the most appropriate drug, or combination of drugs. At present, however, the possibilities for selecting appropriate treatments remain relatively scarce.

\section{The transcriptome of the PDAC correlates with the clinical outcome and could be predictive of drug response}

Progressive accumulation of genetic mutations results in PDAC development, but genetic analysis of the tumors (mutations, amplifications, or deletions) does not help to select specific treatments for patients. This fact indicates that PDAC behavior is regulated at a postgenetic level. In other words, the PDAC phenotype is extremely heterogeneous even though only a small number of mutations are responsible for the heterogeneity. We should therefore assume that these differences are regulated at an epigenetic and transcriptomic level and at a translational and posttranslational level. Therefore, in practical terms, the most appropriate readouts to phenotypically classify a PDAC should be the RNA expression level, or the protein level or posttranslational modifications. To illustrate this statement, some studies that classified PDAC based on their transcriptome $[2,7,21]$ revealed different subtypes that were clinically relevant, and in particular were associated with different clinical outcomes. These studies show that phenotyping PDAC can predict the response to the treatment by immunotherapy [2] and the sensitivity to gemcitabine and erlonitib [7]. In addition, overexpression of CYP3A5 protein is responsible of drug resistance [24] and some post-translational modifications are also associated to chemoresistance [5]. It was in this context our hypothesis emerged: if the transcriptome of tumors is informative about the clinical outcome, it could also be informative about the chemosensitivity of tumoral cells to different drugs. To answer this question, we developed an approach based on the molecular characterization of a significant cohort of pancreatic patients-derived xenograft (PDX) representing almost all PDAC subtypes. Using this approach, we expect to identify molecular signatures.

\section{Patient outcome and transcriptome}

Our preliminary results indicate that the clinical outcome and sensitivity to treatments is associated with a phenotype and is therefore predictable by the molecular analysis of PDAC samples from patients. Our strategy of using a clustering analysis with an unsupervised approach revealed two patient groups characterized by different survival times. Impressively, around 500 transcripts were more represented in short-term survivors, whereas around 400 transcripts were downregulated in longer term survivors. In this cohort, the most differentially expressed genes associated to short-term survival are CALB2, MT1A, 
PTPRZ1, STC1, GSDMC, EMP3, MT1L, KLF12, MCTP1, GNG11, MYBL1, MT1M, ITGA10, FJX1, HTRA1, KRT14, OSBPL6, PTPLA, ANKRD1, VIM whereas genes associated to a longer survival are REG4, CEACAM6, SLC4A4, TM4SF4, CFTR, MUC3A, MUC13, CTSE, PIGR, MUC17, TSPAN8, HSD17B2, PROM1, TM4SF20, ANXA13 and CLDN10. Importantly, with a significant false discovery rate $(>0.001)$, gene ontology analysis on genes that were differentially expressed by these two groups showed a significant enrichment in biological processes associated with cancer, such as the cell cycle, mitosis, response to cellular stresses, DNA metabolism, chromosome organization, and cellular metabolism. This first analysis indicated that these pathways were preferentially modulated between the two patient groups. Analysis of clinical data from both groups of patients showed that patients with short survival had poorly differentiated tumors, whereas patients with longer term survival presented with middle- or well-differentiated tumors [10]. Our data were therefore not so surprising; tumors with lowgrade differentiation are expected to be associated with short-term survival compared to those with well- or middle-grade differentiation [16, 22, 32]. Although the grade of differentiation can be determined microscopically by a pathologist after a pancreatectomy, this is only possible in around $15 \%$ of patients. However, using a set of molecular markers, this could also be estimated in the other $85 \%$ of patients who are biopsied using endoscopic ultrasound (EUS) and fine needle aspiration (FNA), even if only a few cells from the tumor are obtained using this method. In fact, our results suggest that measuring the expression level of some of these molecular indicators in small PDAC samples, as obtained using EUSFNA, would help in predicting PDAC outcome and therefore would help in choosing the best therapeutic strategy.

\section{Chemoresistance and the transcriptome}

In a preliminary study, we analyzed the sensitivity of PDAC primary cell lines to increasing concentrations of five gold-standard chemotherapeutic drugs (gemcitabine, 5FU, oxaliplatin, docetaxel, and the irinotecan active metabolite named SN38) (from 0.001 to $1000 \mu \mathrm{M}$ ). These personalized chemograms allowed us to obtain dose-response curves that characterize tumor chemosensitivity for each patient. Our results demonstrated that each patient-derived cell line has its own chemogram profile, showing a huge heterogeneity in PDAC chemosensitivity [10]. This is clinically relevant, since sensitivity or resistance to one drug predicts neither sensitivity nor resistance to another. Another important point to be noted is that some cells survived, even after 72 hours of incubation with very high concentrations of some drugs, such as with $1000 \mu \mathrm{M}$ of gemcitabine, oxaliplatin, docetaxel, or $5 \mathrm{FU}$, or $100 \mu \mathrm{M}$ of $\mathrm{SN}-38$. This observation can be explained by the fact that primary cultures are representative of the different cell populations present in the tumor; PDAC tumors are known to be heterogeneous [26]. This could be clinically relevant, as the chemogram may detect the percentage of cells that are sensitive or resistant to a drug, and 
therefore may become a helpful tool to the oncologist selecting the second line of treatment for a given patient.

We then studied whether or not there is a correlation between drug response and PDAC phenotype by performing a supervised clustering analysis of the transcriptome. Surprisingly, some sets of genes were identified as being specifically overexpressed or underexpressed in resistant and sensitive cells, supporting the hypothesis that the tumor phenotype determines the response to a treatment. Importantly, a very small number of common genes associated with drug resistance or sensitivity was observed, suggesting that the phenotype of the sensitivity or resistance is specific for each drug. Lastly, another important point is the fact that genes associated with sensitivity or resistance to the drugs are not typically genes associated with survival, indicating that outcome and drug sensitivity are regulated by independent mechanisms.

\section{Repositioning unused drugs for treating PDAC patients is possible}

DNA methyltransferase (DNMT) inhibitors such as 5-AZA-dC demonstrate activity against hematologic malignancies, whereas their efficacy in solid tumors seems to be limited. The rationale for using methyltransferases inhibitors to treat tumors is that neoplastic cells exhibit global hypomethylation with localized hypermethylation of CpG islands and increased levels of methyltransferase activity [12]. Moreover, aberrant hypermethylation of CpG islands is associated with transcriptional silencing of genes, which not only plays a role in tumorigenesis, but may also influence response to anticancer agents [13, 29]. Therefore, reversing gene methylation and epigenetic silencing has the potential to influence tumor growth, sensitivity to anticancer agents, and ultimately clinical outcome [11]. In clinical trials, although 5-AZA-dC has shown an objective response in some patients, its overall efficacy remains relatively low. For these reasons, DNMT inhibitors are not used in the treatment of pancreatic cancers. As a proof-of-concept, we intended to demonstrate that discarded drugs may present a clinical interest for a particular subgroup of patients after identifying the sensitive tumors with specific markers. To this end, we treated several primary cultures of PDAC cancer cells with increasing concentrations of 5-AZA-dC in order to study their sensitivity and to obtain a dose-response curve. Using this approach, we were able to compare these PDAC-derived primary cultures and estimate their relative chemosensitivity. Analysis of the transcriptomes of tumoral cells from sensitive patients reveal that some genes typically expressed in poorly differentiated PDAC, such as MUC3A, MUC5AC, GATA6, or HNF4A, are differentially overexpressed in sensitive PDAC-derived cells compared to resistant cells [15]. These data suggest that 5-AZA-dC treatment is more effective against well- and moderately differentiated tumors than against those that are poorly differentiated.

In the same way, we evaluated the putative use of the FK866 compound, a noncompetitive highly specific inhibitor of NAMPT [25], for treating PDAC. PDAC-derived cells were treated 
with increasing concentrations of FK866 to determine their sensitivity by plotting doseresponse curves. Using this approach, we were able to estimate their relative chemosensitivity by comparing their IC50s. Our data reveal that each PDAC-derived cell culture has its own sensitivity to FK866, with a huge range of IC50s. Next, we hypothesized that NAMPT expression, as a specific target of FK866, could predict drug sensitivity. Consequently, we quantified NAMPT at the transcriptional level in pancreatic PDX. We plotted the mRNA expression level and used IC50 values to reflect and compare the global sensitivity of pancreatic cancer cells; our results indicate that resistance to FK866 positively correlates with the expression level of NAMPT transcripts [3]. In other words, we observed that PDAC expressing lower levels of NAMPT had an increased sensitivity to FK866 treatment.

\section{Identifying novel personalized targets for treating patients with PDAC}

One additional strategy to discover potential markers, and therefore therapeutic targets, for patient stratification is to focus on identifying pathways that are deregulated in tumors, particularly when tumor cell activity absolutely depends on keeping these alterations. It is therefore logical to assume that blocking these pathways with specific inhibitors should lead to cell growth arrest and death, and the consequent tumor regression. Using this rationale, it would be possible to select, by means of a few markers, a particular subgroup of patients whose tumors are "dependent on" a distinct pathway, and use appropriate inhibitors for treating these patients; this is the major goal of modern individualized medicine. In this way, a frequently deregulated although insufficiently therapeutically exploited pathway in PDAC involves "dependence" on the c-Myc oncogene [20]. This transcription factor influences the expression of a significant number of genes involved in cell growth, proliferation, and apoptosis. Consequently, many efforts have been dedicated to identify potent MYC inhibitors as new therapeutic options $[1,14,19,28]$. Key to these efforts have been the discovery of the bromodomain and extraterminal family of proteins (BET), which are efficiently inhibited by BET inhibitors (BETi). Thus, identifying a subgroup of PDAC patients based on their c-MYC status and testing their response to BETi could be of paramount medical importance.

To identify patients with PDAC dependent on MYC activity, we selected a panel of RNAs known to be regulated by MYC and realized a hierarchical clustering on a set of several PDAC transcriptomes. The obtained dendrogram indicates the presence of two clear subgroups, which were defined as MYC-high and MYC-low and that corresponded to around $30 \%$ and $70 \%$ of patients, respectively. We then defined a minimal specific MYC signature of 16 genes to be used to classify tumor subtypes as MYC-high or MYC-low. An independent validation cohort confirmed the efficiency of this minimal signature. We then analyzed whether the subgroup of PDAC with the MYC-high phenotype was more sensitive to pharmacological inhibition of MYC activity, which currently cannot be targeted directly but instead is targeted 
indirectly through the inactivation of BET proteins. To test our hypothesis, we treated a panel of pancreatic PDX with JQ1, a well-characterized BETi. As expected, we found that cells from MYC-high patients (tested in cell culture, spheroids, organoids, and xenografts) exhibit higher sensitivity to BETi treatment than cells from MYC-low patients [4]. Taken together, the in vitro and in vivo results show that MYC-high tumors are more sensitive to BETi. We conclude that it is possible to develop tools to detect tumors with high MYC activity, and can therefore assume that a similar strategy could be used to stratify patients with tumors that are "dependent on" other pathways and enable selection of these patients for novel treatments.

\section{Conclusion and perspectives}

The identification of genetic alterations in PDAC is clearly ineffective to guide a therapeutic approach. To overcome this obstacle, we evaluated the capacity of transcriptomic molecular signatures to identify patients having a particular clinical outcome or a particular pattern of sensitivity to a given treatment (as presented in Figure 1). Whole or partial transcriptomic analysis of PDAC seems to be a promising strategy to reveal the molecular phenotype of this disease. Importantly, testing RNA signatures is easily applicable and of low cost. This is particularly beneficial in non-operable tumors, which represent around $85 \%$ of PDAC. Currently, a EUS-FNA biopsy is taken from these patients to confirm the diagnosis before starting treatment. These biopsies represent a valuable source of cancer cells, which serve as the source of tumor macromolecules such as RNA. In turn, this RNA can be used to measure the expression of particular RNA sets of interest (expression signatures) to define a particular phenotype. Unfortunately, one of the main difficulties with biopsies is that they are typically contaminated by blood, stroma and, in some cases, normal pancreatic or gastrointestinal cells, which may make molecular analyses difficult. The preparation of PDX from biopsies, in order to obtain sufficient clean material, is technically feasible, but it could take up to six months. This time frame is incompatible with a clinical application. The alternative we propose is to prepare organoids directly from these PDAC biopsies, in a time frame of as few as 2-3 weeks. Organoid culturing has been involved in cancer research for the last few years, and has enabled an array of new experimental techniques. This 3D system models the physiology, shape, and dynamics of tumoral cells, producing a relevant and highly adaptable model system. The increased functional relevance of this model compared to the use of $2 \mathrm{D}$ cancer cell lines makes it an invaluable tool for translational research. As the limitations of this system are being overcome to make high-throughput assays possible, it is clear that organoids are becoming a mainstay of cancer research. Pancreatic cancer organoids can be produced from microbiopsies for non-resectable tumors and from macrobiopsies for resectable PDAC. After a few days of growth, this material becomes almost free of stromal, fibrotic, or other contaminants, and can be amplified by culturing. This amplification by cell replication also increases purity, because only epithelial cancerous 
cells will grow in the selective culture media. In practical terms, it is possible to obtain suitable material within 2-3 weeks of culture. It is then easy to purify RNA from organoids and measure the expression of several informative transcripts using an RNA expression platform such as nanoString (Figure 2). This transcriptional level quantification approach has the great advantage of not requiring previous amplification, which can introduce unwanted technical bias. Importantly, all these manipulations take only three additional days. It is very likely that in the near future, the treatment of cancer will be preceded by a precise and extensive molecular characterization of the cancer cells in order to select the most appropriate treatments, creating the long-expected individualized medicine approach for treating patients with PDAC. PDAC is undoubtedly one of the malignant diseases that most urgently needs this type of approach, since treatment with standard drugs is largely ineffective. Although this review has focused on PDAC, a similar strategy could also be applied to other cancer types.

\section{Acknowledgments}

This work was supported by La Ligue Contre le Cancer, INCa, Canceropole PACA, DGOS (labellisation SIRIC), and INSERM.

\section{References}

[1] D. Annibali, J.R. Whitfield, E. Favuzzi, T. Jauset, E. Serrano, I. Cuartas, S. Redondo-Campos, G. Folch, A. Gonzalez-Junca, N.M. Sodir, D. Masso-Valles, M.E. Beaulieu, L.B. Swigart, M.M. Mc Gee, M.P. Somma, S. Nasi, J. Seoane, G.I. Evan, L. Soucek, Myc inhibition is effective against glioma and reveals a role for Myc in proficient mitosis, Nature communications, 5 (2014) 4632.

[2] P. Bailey, D.K. Chang, K. Nones, A.L. Johns, A.M. Patch, M.C. Gingras, D.K. Miller, A.N. Christ, T.J. Bruxner, M.C. Quinn, C. Nourse, L.C. Murtaugh, I. Harliwong, S. Idrisoglu, S. Manning, E. Nourbakhsh, S. Wani, L. Fink, O. Holmes, V. Chin, M.J. Anderson, S. Kazakoff, C. Leonard, F. Newell, N. Waddell, S. Wood, Q. Xu, P.J. Wilson, N. Cloonan, K.S. Kassahn, D. Taylor, K. Quek, A. Robertson, L. Pantano, L. Mincarelli, L.N. Sanchez, L. Evers, J. Wu, M. Pinese, M.J. Cowley, M.D. Jones, E.K. Colvin, A.M. Nagrial, E.S. Humphrey, L.A. Chantrill, A. Mawson, J. Humphris, A. Chou, M. Pajic, C.J. Scarlett, A.V. Pinho, M. Giry-Laterriere, I. Rooman, J.S. Samra, J.G. Kench, J.A. Lovell, N.D. Merrett, C.W. Toon, K. Epari, N.Q. Nguyen, A. Barbour, N. Zeps, K. Moran-Jones, N.B. Jamieson, J.S. Graham, F. Duthie, K. Oien, J. Hair, R. Grutzmann, A. Maitra, C.A. lacobuzio-Donahue, C.L. Wolfgang, R.A. Morgan, R.T. Lawlor, V. Corbo, C. Bassi, B. Rusev, P. Capelli, R. Salvia, G. Tortora, D. Mukhopadhyay, G.M. Petersen, I. Australian Pancreatic Cancer Genome, D.M. Munzy, W.E. Fisher, S.A. Karim, J.R. Eshleman, R.H. Hruban, C. Pilarsky, J.P. Morton, O.J. Sansom, A. Scarpa, E.A. Musgrove, U.M. Bailey, O. Hofmann, R.L. Sutherland, D.A. Wheeler, A.J. Gill, R.A. Gibbs, J.V. Pearson, N. Waddell, A.V. Biankin, S.M. Grimmond, Genomic analyses identify molecular subtypes of pancreatic cancer, Nature, 531 (2016) 47-52.

[3] M. Barraud, J. Garnier, C. Loncle, O. Gayet, C. Lequeue, S. Vasseur, B. Bian, P. Duconseil, M. Gilabert, M. Bigonnet, A. Maignan, V. Moutardier, S. Garcia, O. Turrini, J.R. Delpero, M. Giovannini, P. Grandval, M. Gasmi, M. Ouaissi, V. Secq, F. Poizat, N. Guibert, J. lovanna, N. Dusetti, A pancreatic 
ductal adenocarcinoma subpopulation is sensitive to FK866, an inhibitor of NAMPT, Oncotarget, 7 (2016) 53783-53796.

[4] B. Bian, M. Bigonnet, O. Gayet, C. Loncle, A. Maignan, M. Gilabert, V. Moutardier, S. Garcia, O. Turrini, J.R. Delpero, M. Giovannini, P. Grandval, M. Gasmi, M. Ouaissi, V. Secq, F. Poizat, R. Nicolle, Y. Blum, L. Marisa, M. Rubis, J.L. Raoul, J.E. Bradner, J. Qi, G. Lomberk, R. Urrutia, A. Saul, N. Dusetti, J. lovanna, Gene expression profiling of patient-derived pancreatic cancer xenografts predicts sensitivity to the BET bromodomain inhibitor JQ1: implications for individualized medicine efforts, EMBO molecular medicine, 9 (2017) 482-497.

[5] T. Bonacci, S. Audebert, L. Camoin, E. Baudelet, G. Bidaut, M. Garcia, Witzel, II, N.D. Perkins, J.P. Borg, J.L. Iovanna, P. Soubeyran, Identification of new mechanisms of cellular response to chemotherapy by tracking changes in post-translational modifications by ubiquitin and ubiquitin-like proteins, Journal of proteome research, 13 (2014) 2478-2494.

[6] H.A. Burris, 3rd, M.J. Moore, J. Andersen, M.R. Green, M.L. Rothenberg, M.R. Modiano, M.C. Cripps, R.K. Portenoy, A.M. Storniolo, P. Tarassoff, R. Nelson, F.A. Dorr, C.D. Stephens, D.D. Von Hoff, Improvements in survival and clinical benefit with gemcitabine as first-line therapy for patients with advanced pancreas cancer: a randomized trial, Journal of clinical oncology : official journal of the American Society of Clinical Oncology, 15 (1997) 2403-2413.

[7] E.A. Collisson, A. Sadanandam, P. Olson, W.J. Gibb, M. Truitt, S. Gu, J. Cooc, J. Weinkle, G.E. Kim, L. Jakkula, H.S. Feiler, A.H. Ko, A.B. Olshen, K.L. Danenberg, M.A. Tempero, P.T. Spellman, D. Hanahan, J.W. Gray, Subtypes of pancreatic ductal adenocarcinoma and their differing responses to therapy, Nature medicine, 17 (2011) 500-503.

[8] T. Conroy, F. Desseigne, M. Ychou, O. Bouche, R. Guimbaud, Y. Becouarn, A. Adenis, J.L. Raoul, S. Gourgou-Bourgade, C. de la Fouchardiere, J. Bennouna, J.B. Bachet, F. Khemissa-Akouz, D. PereVerge, C. Delbaldo, E. Assenat, B. Chauffert, P. Michel, C. Montoto-Grillot, M. Ducreux, U. Groupe Tumeurs Digestives of, P. Intergroup, FOLFIRINOX versus gemcitabine for metastatic pancreatic cancer, The New England journal of medicine, 364 (2011) 1817-1825.

[9] E. Costello, W. Greenhalf, J.P. Neoptolemos, New biomarkers and targets in pancreatic cancer and their application to treatment, Nature reviews. Gastroenterology \& hepatology, 9 (2012) 435-444.

[10] P. Duconseil, M. Gilabert, O. Gayet, C. Loncle, V. Moutardier, O. Turrini, E. Calvo, J. Ewald, M. Giovannini, M. Gasmi, E. Bories, M. Barthet, M. Ouaissi, A. Goncalves, F. Poizat, J.L. Raoul, V. Secq, S. Garcia, P. Viens, J. lovanna, N. Dusetti, Transcriptomic analysis predicts survival and sensitivity to anticancer drugs of patients with a pancreatic adenocarcinoma, The American journal of pathology, 185 (2015) 1022-1032.

[11] G. Egger, G. Liang, A. Aparicio, P.A. Jones, Epigenetics in human disease and prospects for epigenetic therapy, Nature, 429 (2004) 457-463.

[12] M. Ehrlich, Cancer-linked DNA hypomethylation and its relationship to hypermethylation, Current topics in microbiology and immunology, 310 (2006) 251-274.

[13] M. Esteller, Relevance of DNA methylation in the management of cancer, The Lancet. Oncology, 4 (2003) 351-358.

[14] S. Fletcher, E.V. Prochownik, Small-molecule inhibitors of the Myc oncoprotein, Biochimica et biophysica acta, 1849 (2015) 525-543.

[15] O. Gayet, C. Loncle, P. Duconseil, M. Gilabert, M.B. Lopez, V. Moutardier, O. Turrini, E. Calvo, J. Ewald, M. Giovannini, M. Gasmi, E. Bories, M. Barthet, M. Ouaissi, A. Goncalves, F. Poizat, J.L. Raoul, V. Secq, S. Garcia, P. Viens, N. Dusetti, J. lovanna, A subgroup of pancreatic adenocarcinoma is sensitive to the 5-aza-dC DNA methyltransferase inhibitor, Oncotarget, 6 (2015) 746-754.

[16] R.J. Geer, M.F. Brennan, Prognostic indicators for survival after resection of pancreatic adenocarcinoma, American journal of surgery, 165 (1993) 68-72; discussion 72-63.

[17] J. lovanna, M.C. Mallmann, A. Goncalves, O. Turrini, J.C. Dagorn, Current knowledge on pancreatic cancer, Frontiers in oncology, 2 (2012) 6.

[18] H.L. Kindler, T. loka, D.J. Richel, J. Bennouna, R. Letourneau, T. Okusaka, A. Funakoshi, J. Furuse, Y.S. Park, S. Ohkawa, G.M. Springett, H.S. Wasan, P.C. Trask, P. Bycott, A.D. Ricart, S. Kim, E. Van Cutsem, Axitinib plus gemcitabine versus placebo plus gemcitabine in patients with advanced 
pancreatic adenocarcinoma: a double-blind randomised phase 3 study, The Lancet. Oncology, 12 (2011) 256-262.

[19] M.R. McKeown, J.E. Bradner, Therapeutic strategies to inhibit MYC, Cold Spring Harbor perspectives in medicine, 4 (2014).

[20] J.A. Mertz, A.R. Conery, B.M. Bryant, P. Sandy, S. Balasubramanian, D.A. Mele, L. Bergeron, R.J. Sims, 3rd, Targeting MYC dependence in cancer by inhibiting BET bromodomains, Proceedings of the National Academy of Sciences of the United States of America, 108 (2011) 16669-16674.

[21] R.A. Moffitt, R. Marayati, E.L. Flate, K.E. Volmar, S.G. Loeza, K.A. Hoadley, N.U. Rashid, L.A. Williams, S.C. Eaton, A.H. Chung, J.K. Smyla, J.M. Anderson, H.J. Kim, D.J. Bentrem, M.S. Talamonti, C.A. lacobuzio-Donahue, M.A. Hollingsworth, J.J. Yeh, Virtual microdissection identifies distinct tumor- and stroma-specific subtypes of pancreatic ductal adenocarcinoma, Nature genetics, 47 (2015) 1168-1178.

[22] H.J. Moon, J.Y. An, J.S. Heo, S.H. Choi, J.W. Joh, Y.I. Kim, Predicting survival after surgical resection for pancreatic ductal adenocarcinoma, Pancreas, 32 (2006) 37-43.

[23] M.J. Moore, D. Goldstein, J. Hamm, A. Figer, J.R. Hecht, S. Gallinger, H.J. Au, P. Murawa, D. Walde, R.A. Wolff, D. Campos, R. Lim, K. Ding, G. Clark, T. Voskoglou-Nomikos, M. Ptasynski, W. Parulekar, G. National Cancer Institute of Canada Clinical Trials, Erlotinib plus gemcitabine compared with gemcitabine alone in patients with advanced pancreatic cancer: a phase III trial of the National Cancer Institute of Canada Clinical Trials Group, Journal of clinical oncology : official journal of the American Society of Clinical Oncology, 25 (2007) 1960-1966.

[24] E.M. Noll, C. Eisen, A. Stenzinger, E. Espinet, A. Muckenhuber, C. Klein, V. Vogel, B. Klaus, W. Nadler, C. Rosli, C. Lutz, M. Kulke, J. Engelhardt, F.M. Zickgraf, O. Espinosa, M. Schlesner, X. Jiang, A. Kopp-Schneider, P. Neuhaus, M. Bahra, B.V. Sinn, R. Eils, N.A. Giese, T. Hackert, O. Strobel, J. Werner, M.W. Buchler, W. Weichert, A. Trumpp, M.R. Sprick, CYP3A5 mediates basal and acquired therapy resistance in different subtypes of pancreatic ductal adenocarcinoma, Nature medicine, 22 (2016) 278-287.

[25] U.H. Olesen, M.K. Christensen, F. Bjorkling, M. Jaattela, P.B. Jensen, M. Sehested, S.J. Nielsen, Anticancer agent CHS-828 inhibits cellular synthesis of NAD, Biochemical and biophysical research communications, 367 (2008) 799-804.

[26] V.R. Penchev, Z.A. Rasheed, A. Maitra, W. Matsui, Heterogeneity and targeting of pancreatic cancer stem cells, Clinical cancer research : an official journal of the American Association for Cancer Research, 18 (2012) 4277-4284.

[27] R.L. Siegel, K.D. Miller, A. Jemal, Cancer statistics, 2016, CA: a cancer journal for clinicians, 66 (2016) 7-30.

[28] L. Soucek, J. Whitfield, C.P. Martins, A.J. Finch, D.J. Murphy, N.M. Sodir, A.N. Karnezis, L.B. Swigart, S. Nasi, G.I. Evan, Modelling Myc inhibition as a cancer therapy, Nature, 455 (2008) 679-683. [29] J.M. Teodoridis, G. Strathdee, R. Brown, Epigenetic silencing mediated by CpG island methylation: potential as a therapeutic target and as a biomarker, Drug resistance updates : reviews and commentaries in antimicrobial and anticancer chemotherapy, 7 (2004) 267-278.

[30] E. Van Cutsem, W.L. Vervenne, J. Bennouna, Y. Humblet, S. Gill, J.L. Van Laethem, C. Verslype, W. Scheithauer, A. Shang, J. Cosaert, M.J. Moore, Phase III trial of bevacizumab in combination with gemcitabine and erlotinib in patients with metastatic pancreatic cancer, Journal of clinical oncology : official journal of the American Society of Clinical Oncology, 27 (2009) 2231-2237.

[31] A. Vincent, J. Herman, R. Schulick, R.H. Hruban, M. Goggins, Pancreatic cancer, Lancet, 378 (2011) 607-620.

[32] D.D. You, H.G. Lee, J.S. Heo, S.H. Choi, D.W. Choi, Prognostic factors and adjuvant chemoradiation therapy after pancreaticoduodenectomy for pancreatic adenocarcinoma, Journal of gastrointestinal surgery : official journal of the Society for Surgery of the Alimentary Tract, 13 (2009) 1699-1706. 


\section{Figures legend}

\section{Figure 1}

Strategy to selecting specific markers of sensitivity to a cytotoxic treatment. A cohort of PDAC-derived cells are treated with a given cytotoxic compound to determine their sensitivity. Expression of these PDAC was determined by RNA-seq and the bioinformatics analysis identifies differentially expressed genes between PDAC sensitive and PDAC resistant as candidate markers to be used as indicators. These markers are confirmed in an independent (validation) cohort of PDAC before their utilization in clinical trials.

\section{Figure 2}

Molecular signatures will be obtained for individualized treatment of patients with PDAC in the near future. Molecular signatures should be obtained for patients with operable PDAC (15\% of cases), but will be of particular interest for patients with non-operable PDAC (85\% of cases). These samples represent a valuable source of cancer cells that serve as the source of RNA. The material obtained is largely contaminated by blood and tumor stroma or neighboring tissues. The alternative strategy is to prepare organoids directly from surgical samples or biopsies. This allows amplification by cell replication, and purity, since only epithelial cancerous cells grow in the selective culture media. Sufficient organoids can be obtained within 2-3 weeks of culture. RNA from organoids can easily be purified, and the expression of several informative transcripts can be measured by a nanoString platform in 23 additional days. 


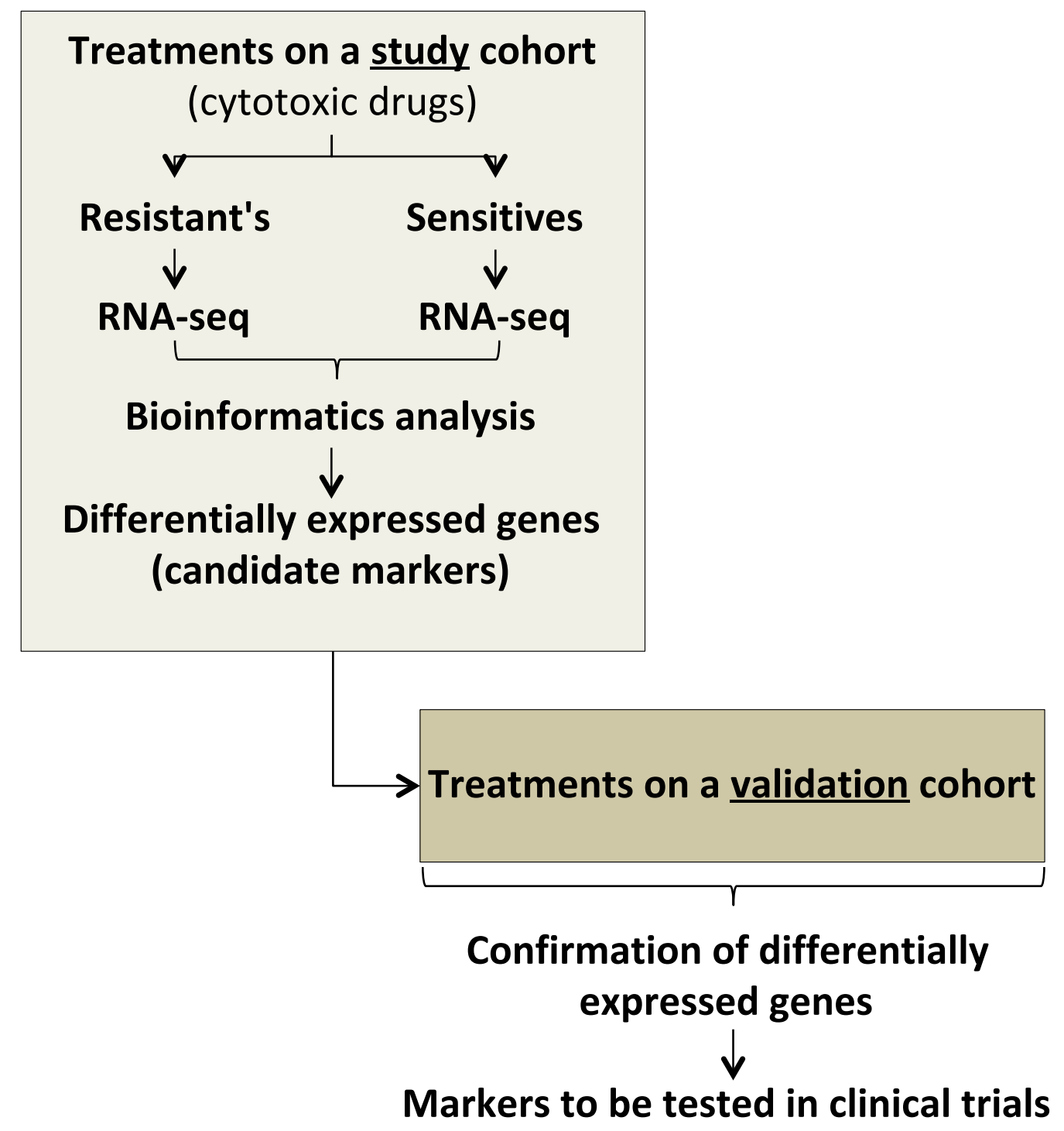

Figure 1 


\section{2-3 weeks}

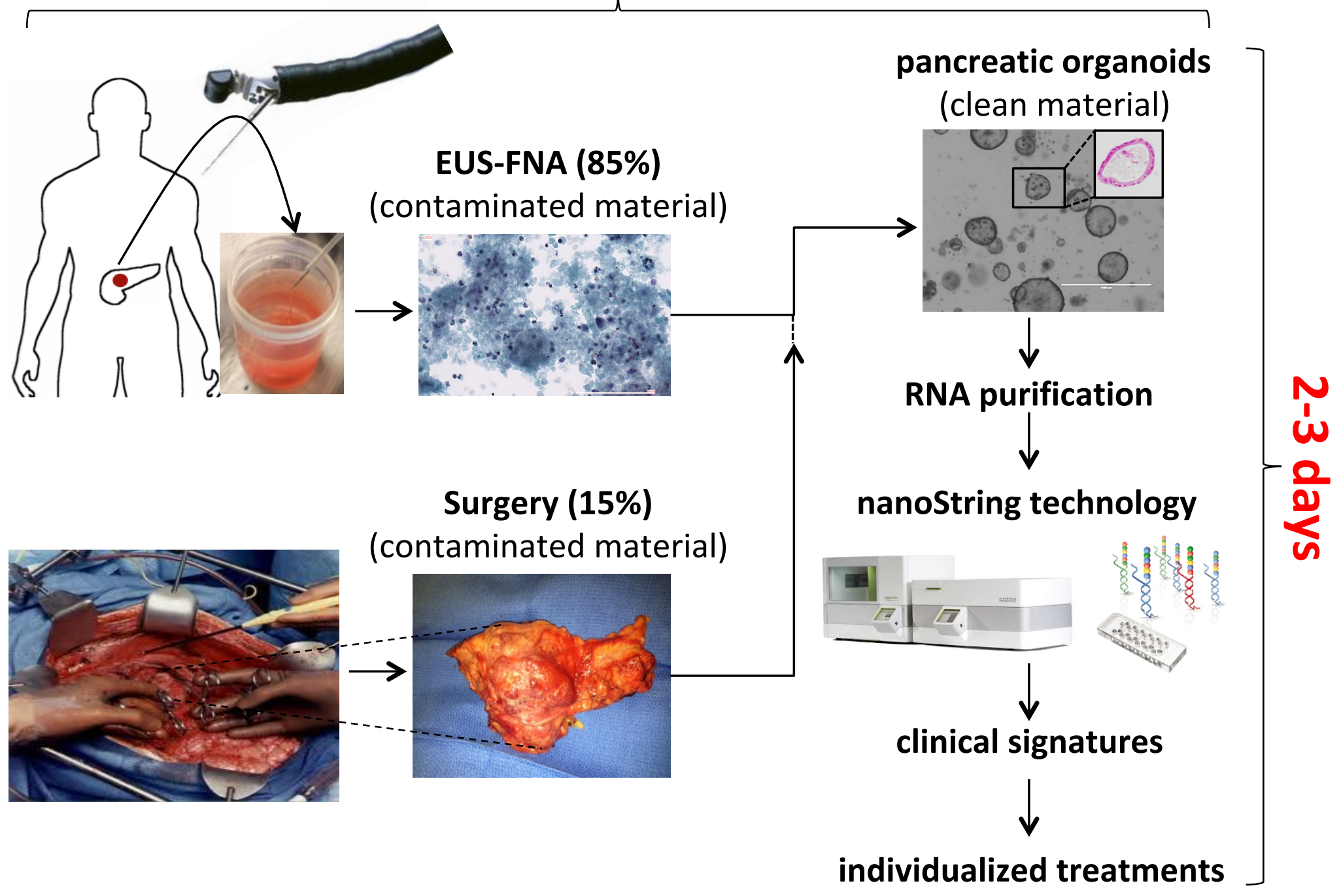

Figure 2 